\title{
Effects of the electronic structure on the dc conductance of Fibonacci superlattices
}

\author{
Enrique Maciä* and Francisco Domínguez-Adame \\ Departamento de Fŕsica de Materiales, Facultad de Físicas, Universidad Complutense, E-28040 Madrid, Spain \\ Angel Sánchez \\ Escuela Politécnica Superior, Universidad Carlos III de Madrid, c./Butarque 15, E-28911 Leganés, Madrid, Spain
}

(Received 1 September 1993; revised manuscript received 16 December 1993)

\begin{abstract}
We derive a discrete Hamiltonian describing a Fibonacci superlattice in which the electronic potential is taken to be an array of equally spaced $\delta$ potentials, whose strengths modulate the chemical composition in the growth direction. In this model both diagonal and off-diagonal elements of the Hamiltonian matrix become mutually related through the potential strengths. The corresponding energy spectrum and related magnitudes, such as the Lyapunov coefficient, transmission coefficient, and Landauer resistance, exhibit a highly fragmented, self-similar nature. We investigate the influence of the underlying spectrum structure on the de conductance at different temperatures obtaining analytical expressions which relate special features of the dc conductance with certain parameters that characterize the electronic spectrum of Fibonacci superlattices.
\end{abstract}

\section{INTRODUCTION}

The rapid progress achieved with crystal growth technologies, like molecular beam epitaxy, has made it possible to grow artificial structures with periodic or aperiodic modulation of chemical composition along the growth direction. In this way, the one-dimensional (ID) ordering introduced in the manufacturing process gives rise to novel physical properties such as the formation of minibands which reflect the long-range, quantum correlation present in multilayered superstructures. In fact, the electronic properties of superlattices are determined both by the chemical nature of the constituent bulk materials as well as the layer thicknesses so that these structures can be grown to tailor their electronic properties as required. On the other side, the discovery of quasicrystals has attracted strong interest in the physical properties of quasiperiodic systems, and numerous theoretical works have been focused on the electronic properties of iD specific realizations. ${ }^{1-4}$ In these systems quasiperiodicity leads to higly fragmented spectra that are Cantor sets having pure-point, absolutely continuous, and singular continuous components which respectively determine the existence of localized, extended, and critical states. In some cases, quasiperiodic potentials admit the existence of a mobility edge and allow for a metal-insulator transition under appropriate conditions. ${ }^{5,6}$ Therefore, it seems natural to consider the transport properties which could appear in quasiperiodically modulated superlattices.

Following the first fabrication of a quasiperiodic semiconductor superlattice by Merlin and co-workers ${ }^{7,8}$ most works have considered the Fibonacci sequence as a typical example of a quasiperiodic system. However, we think it has not properly been stressed in the literature that systems ordered according to the Fibonacci sequence exhibit some characteristic properties which are not shared by other quasiperiodic modulations. In fact, Fibonacci arrangements show spectra with a hierarchy of splitting minibands displaying self-similar patterns. This point has been experimentally observed in a variety of situations including electronic, ${ }^{9}$ phonon, ${ }^{10}$ third-sound transmission, ${ }^{11}$ and absorption spectra with magnetic fields ${ }^{12}$ for different Fibonacci systems. Furthermore, theoretical calculations indicate that almost all electronic eigenstates are neither extended nor localized but critical in a Fibonacci lattice. ${ }^{13}$ Similar results also apply to the plasmon spectrum of Fibonacci semiconductor superlattices. ${ }^{14}$ In addition, no evidence of mobility edges has been found, in contrast to what has been reported for other, non-Fibonaccian, quasiperiodic lattices. ${ }^{15}$ All these considerations clearly indicate that the study of Fibonacci superlattices (FSL's) is interesting in its own right, since they are ideally suited for the understanding of the physical nature of critical states. Furthermore, as far as experimental studies are concerned, we must remark on the fact that FSL properties are robust, i.e., may be preserved in the presence of significant levels of randomness. In fact, $\mathrm{x}$-ray diffraction studies show that moderately large growth fluctuations in the sequential deposition of layers do not disturb seriously the quasiperiodic order exhibited by FSL's. ${ }^{8}$

Recent investigations on perpendicular transport properties of photoexcited carriers in semiconductor FSL's revealed the existence of a self-similar structure in the energy spectrum with localization properties somewhat intermediate between periodic and random systems. ${ }^{16}$ Moreover, small oscillations of the resistance of a FSL under a fixed magnetic field have been reported very recently. ${ }^{17}$ The peak positions of the measured resistance are in good agreement with those of the transmission gaps predicted in simple theoretical models, indicating a welldefined self-similar scheme. These results clearly renew 
the interest in the peculiar transport properties through Fibonacci systems.

The electronic spectrum structure of FSL's, within the tight-binding, single-band approximation, is firmly established for two special cases. These correspond to the situation in which we choose the (say, GaAs) wells to have all the same width but the barriers (say, GaAlAs) to have either different widths (transfer models) or different heights (on-site models). In these tight-binding analyses the multilayered heterostructures can be identified with a series of quantum wells which are coupled by a nearly constant interaction energy. Less attention has been paid to the class of models for which both diagonal and off-diagonal terms are present in the Hamiltonian describing the interaction between nearest-neighbor quantum wells. ${ }^{13,18}$ These mixed models are more appropriate in order to describe realistic FSL's in which the hopping terms would take different values depending upon the chemical nature of the layers.

The aim of this paper is to uncover the relationship between the transport properties of a Fibonacci system and the structure of its underlying energy spectrum. To this end we will work out a general treatment, within the tight-binding approximation, which will allow us to obtain the electronic spectrum of a system describing a FSL in which specific interactions between nearest-neighbor building blocks are taken into account.

The rest of the paper is planned as follows. The major features of our model are presented at the beginning of Sec. II. Afterward, the one-electron Schrödinger equation describing the electron dynamics is reduced to an appropiate tight-binding form. We solve this equation by means of standard transfer-matrix techniques and discuss the resulting energy spectrum in Sec. III. Next, we turn to our main issue: the conductance properties of the superlattice. In Sec. IV we present the conductance behavior at zero temperature and relate the fractal structure of the spectrum with the presence of strong conductance fluctuations. In Sec. $V$ we take into account finitetemperature effects in the dc conductance. Through this section interesting features appearing in the conductance at low temperatures are described and its relationship with the fragmented nature of the electronic spectrum discussed. We close Sec. V with the study of the hightemperature limit where we find a power-law scaling of conductance with system size. Section VI contains final considerations as well as possible applications to a number of physical contexts.

\section{DESCRIPTION OF THE MODEL}

Before entering into the description of the model itself, some words are in order regarding its physical relevance and its applicability. To grow a FSL we must define two distinct building blocks, say $A$ and $B$, and order them according to the Fibonacci sequence. Each building block can be composed of one or more layers of different materials and can have arbitrary thicknesses. A Fibonacci sequence $S_{n}$ of order $n$ is obtained by $n$ succesive applications of the transformation $A \rightarrow A B$ and $B \rightarrow A$. The sequence $S_{n}$ comprises $F_{n}$ elements $A$ and $F_{n-1}$ elements $B, F_{n}$ being the $n$th Fibonacci number given by the recurrent law $F_{n}=F_{n-1}+F_{n-2}$ with $F_{0}=F_{1}=1$. As $n$ increases the ratio $F_{n-1} / F_{n}$ converges toward $\tau=(\sqrt{5}-1) / 2$ which is known as the inverse golden mean. For the sake of generality we do not consider any specific potential shape associated with the $A$ and $B$ building blocks composing our FSL, since, at present, there exists a number of different potential profiles (square barriers, V-shaped, sawtooth, parabolic) for multilayered structures. Instead, we shall assume a quite general expression for the block potentials given by means of point interaction potentials. The term point interaction potential refers to any arbitrary sharply peaked potential approaching the $\delta$-function limit (zero width and constant area). Such potentials are often used in some physical contexts in solid state physics ${ }^{18}$ since, with limitations, they are good candidates to replace actual, short-ranged, 1D potentials. ${ }^{20}$

Let us consider a FSL with $N \equiv F_{n}$ building blocks. Since we are dealing with a chemically modulated quasiperiodic structure, we will take equally spaced blocks defining a periodic array of period $d$ and we introduce the quasiperiodic modulation by means of an appropiate choice of their chemical composition. The dynamics of electrons in this superstructure will be described by the following Schrödinger equation in units such that $\hbar=m^{*}=1$ :

$$
\left[-\frac{1}{2} \frac{d^{2}}{d x^{2}}-\sum_{n} \lambda_{n} \delta(x-n d)\right] \psi(x)=E \psi(x)
$$

where we allow the potential strength $\lambda_{n}$ to take on two values, $\lambda_{A}$ and $\lambda_{B}$, arranged according to the Fibonacci sequence. Hereafter we restrict ourselves to attractive potentials $\left(\lambda_{n}>0\right)$ and take $\lambda_{A}=1$ without loss of generality. We express the electron wave function as a linear combination of localized orbitals $\psi(x)=\sum_{n} C_{n} \phi_{n}(x-$ $n d)$, where $\phi_{n}(x-n d)=\sqrt{\lambda_{n}} \exp \left(-\lambda_{n}|x-n d|\right)$ is the normalized eigenfunction of a $\delta$ function placed at $x=n d$. Neglecting the overlap between different orbitals and assuming that only nearest-neighbor interactions are significant, we obtain the following tight-binding equation for the amplitudes:

$$
\left(E-\epsilon_{n}\right) C_{n}=t_{n, n+1} C_{n+1}+t_{n, n-1} C_{n-1}
$$

where the on-site energies and the hopping integrals are mutually related by the expressions

$$
\begin{aligned}
\epsilon_{n} & =-\frac{1}{2} \lambda_{n}^{2} \\
t_{n, n \pm 1} & =-\sqrt{\lambda_{n}^{3} \lambda_{n \pm 1}} \exp \left(-\lambda_{n \pm 1} d\right)
\end{aligned}
$$

Thus, in our model the chemical modulation parameter $\lambda_{n}$ determines both the on-site energies and the hopping integrals describing the coupling between nearest building blocks. Equation (2) can be cast into the matrix form 


$$
\begin{aligned}
\left(\begin{array}{c}
C_{k-1} \\
C_{k}
\end{array}\right) & =\left(\begin{array}{cc}
\frac{E-\epsilon_{k}}{t_{k, k-1}} & -\frac{t_{k, k+1}}{t_{k, k-1}}- \\
1 & 0
\end{array}\right)\left(\begin{array}{c}
C_{k} \\
C_{k+1}
\end{array}\right) \\
& \equiv M_{k}\left(\begin{array}{c}
C_{k} \\
C_{k+1}
\end{array}\right),
\end{aligned}
$$

where $E$ is the electron energy. The solution of the dynamical equation (5) is obtained by using standard transfer-matrix techniques. To minimize end effects we take periodic boundary conditions. Hence, the allowed regions of the electronic spectrum can be numerically determined from the usual condition

$$
|\operatorname{Tr} M(N)| \equiv\left|\operatorname{Tr}\left(\prod_{k=1}^{N} M_{k}\right)\right| \leq 2 .
$$

We address this calculation in the next section.

\section{ELECTRONIC STRUCTURE OF THE FIBONACCI SUPERLATTICE}

In our numerical simulations, we have studied in detail different realizations of the FSL by varying the sample length $N$, the period $d$, and the ratio $\alpha \equiv \lambda_{B} / \lambda_{A}$ which accounts for the chemical diversity of the superlattice. The more distant $\alpha$ from unity, the more different the chemical nature of the two building blocks. We set parameter ranges given by $8 \leq N \leq 1597,0.05 \leq \alpha \leq 3$, and $I \leq d \leq 6$. In all cases considered we have observed a tetrafurcation pattern of the energy spectrum, characterized by the presence of four main subbands separated by well-defined gaps. Inside each main subband the fragmentation scheme follows a trifurcation pattern in which each subband further trifurcates obeying a hierarchy of splitting from one to three subsubbands. These results are illustrated in Fig. 1. In this figure we show the behavior of the Lyapunov coefficient given by the

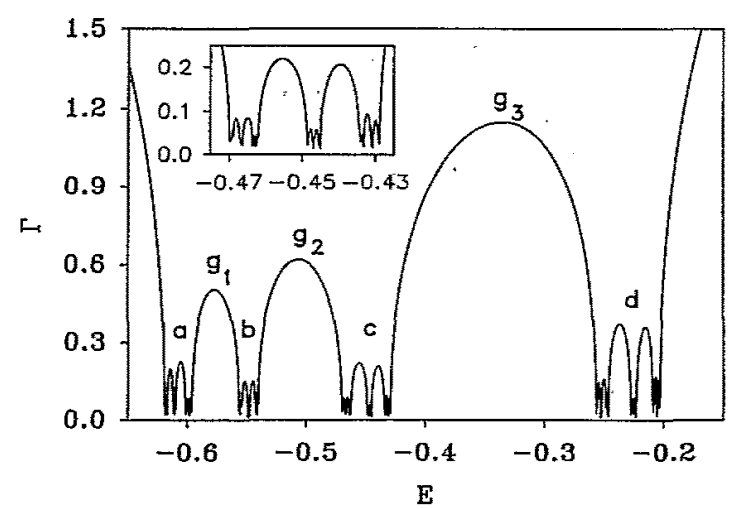

FIG. 1. Lyapunov coefficient versus energy plot for system parameters $\alpha=0.75, d=2.5$, and $N=987$. The labels $a, b$, $c$, and $d$ denote the main subbands appearing at the first level of fragmentation of the spectrum. Correspondingly, the labels $g_{1}, g_{2}, g_{3}$ indicate the position of the main gaps. The global tetrafurcation of the spectrum is clearly visible from this plot as well as its self-similar character, displayed in more detail in the inset. expression $\Gamma=\left(M_{11}^{2}+M_{12}^{2}+M_{21}^{2}+M_{22}^{2}\right) / N$ where $M_{i j}$ stand for the elements of matrix $M(N) .{ }^{21}$ The four main subbands, labeled $a, b, c$, and $d$, are separated by well-developed local maxima of the Lyapunov coefficient which define the main gaps positions $g_{1}, g_{2}$, and $g_{3}$. In the inset an enlarged view of the centralmost main subband $c$ shows the self-similar nature of the spectrum structure. It is well known that the total number of subbands composing the fragmented spectrum of a FSL of length $F_{n}$ is just given by the number of building blocks present in it. ${ }^{22}$ In addition, we have observed that the number of subsubbands in each main cluster is given by $N_{a}=N_{c}=F_{n-3}, N_{b}=F_{n-4}^{2}, N_{d}=F_{n-2}$. A similar distribution rule has been reported for on-site models. ${ }^{23}$

In our numerical study we have analyzed the dependence of the relative widths of both main subbands and gaps with the model parameters $\alpha$ and $N$. In the first place we consider the dependence of the energy spectrum structure on the sample length. Since, strictly speaking, quasiperiodicity is only observable in the limit $N \rightarrow \infty$, our results provide information on the prefractal signature of the FSL. We have observed that both the position and widths of the main subbands of the spectrum converge very rapidly to stable values with increasing sample size for any fixed value of the parameter $\alpha$. We shall refer to this behavior as asymptotic stability of the spectrum; it implies that its global structure can be obtained in practice by considering very short approximants to the infinite quasiperiodic chain (as short as $55=F_{10}$ units). This remarkable behavior suggests that the first stage of the spectrum hierarchical splitting is mainly determined by short-range effects, in agreement with real space renormalization group ideas where the number of energy levels appearing at the first stage of the renormalization process determines the number of main clusters in the spectrum. ${ }^{24,25}$

Next we consider the dependence of the main features of the spectrum on the chemical diversity parameter $\alpha$. Our results are plotted in Fig. 2. As the chemical diversity of the lattice increases, the main subbands become progresively narrower [Fig. 2(a)] and the gaps steadily wider [Fig. 2(b)]. The behavior of the normalized widths is clearly nonlinear with $\alpha$. Another interesting feature shown in Fig. 2 concerns the existence of crossing points in the bandwidth evolution. The existence of such points implies that the relative importance of different subbands and gaps appearing in the spectrum can be accurately controlled by an appropiate choice of the chemical composition of the sample. Thus, for $\alpha=0.95$ we have a global pattern spectrum in which $b>a, g_{2}>g_{3}$, and $c>d$ whereas for $\alpha=0.7$ the situation is completely reversed. Finally, we have calculated the normalized equivalent bandwidth of the spectrum $S$, defined as the ratio between the sum of all the allowed energy intervals and the bandwidth of a periodic lattice made of identical $A$ blocks. As can be expected from the Cantor-like nature of Fibonaccian spectra, the equivalent bandwidth vanishes as the system size grows. Moreover we have obtained that $S$ varies as the square root of $N$ for large enough $N$ obeying a decay law which can be approximately written as 


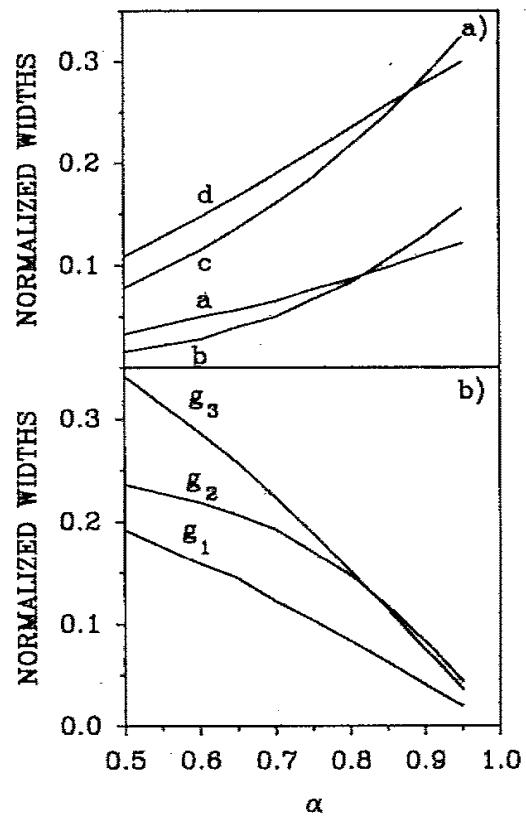

FIG. 2. Dependence of the main (a) subbands and (b) gaps normalized width on the chemical composition of the sample. The width of each spectrum feature is normalized to the bandwidth corresponding to the perfect $A$ superlattice. The labels appearing on each curve correspond to those given in Fig. 1.

$$
\ln S=-(1-\alpha)(4.3+\ln \sqrt{N}) .
$$

Therefore, for a given value of the chemical diversity parameter, we obtain a linear evolution of the equivalent bandwidth in a $\ln S$ versus $\ln N$ plot. According with earlier works ${ }^{1,14}$ such a behavior is characteristic of a singular continuous spectrum signature for which all the wave functions are critical, i.e., neither localized nor extended in a standard way. Since this fact becomes independent of the superlattice chemical composition, we can conclude that a metal-insulator transition depending on a suitable choice of the chemical composition is not to be expected in our model.

\section{CONDUCTANCE OF THE FIBONACCI SUPERLATTICE AT ZERO TEMPERATURE}

The richness in structure displayed by the electronic spectrum of a FSL should be reflected, to some extent, in its transport properties, and evidence for this to be the case has recently been reported in experimental works. ${ }^{9,16,17}$ Generally speaking there are two factors which must be taken into account in order to evaluate the relative importance of typical quasiperiodic effects on the perpendicular transport of FSL's. On the one hand, since these effects are esentially quantum in nature, we must consider systems with strong coupling between adjacent blocks. In our model the degree of coupling between nearest-neighbor blocks is given by the parameter $d$. We have numerically checked that the overall features of the electronic spectrum are rather insensitive to the adopted value of this parameter provided that $d<3$. In our units this condition is fulfilled by GaAs-GaAlAs superlattices (electron effective mass $m^{*}=0.067$ ) with periods ranging from $70 \AA$ to $340 \AA$ and height barriers in the interval 4-100 meV, respectively. In order to obtain general results from now on we shall take $d=2.5$ as a representative value for most of the FSL realizations discussed in the literature. On the other hand, we should consider electron-phonon scattering effects which tend to disrupt coherent quantum transport. These effects crucially depend on the sample temperature and it may be confidently expected that their influence can be neglected at very low temperatures. In this section we shall consider electron propagation through a FSL at zero temperature. In this case the relationship between the electrical conductance at zero temperature and the transmission coefficient, $\tau(E)$, is given by the well-known dimensionless single-channel Landauer formula ${ }^{26}$
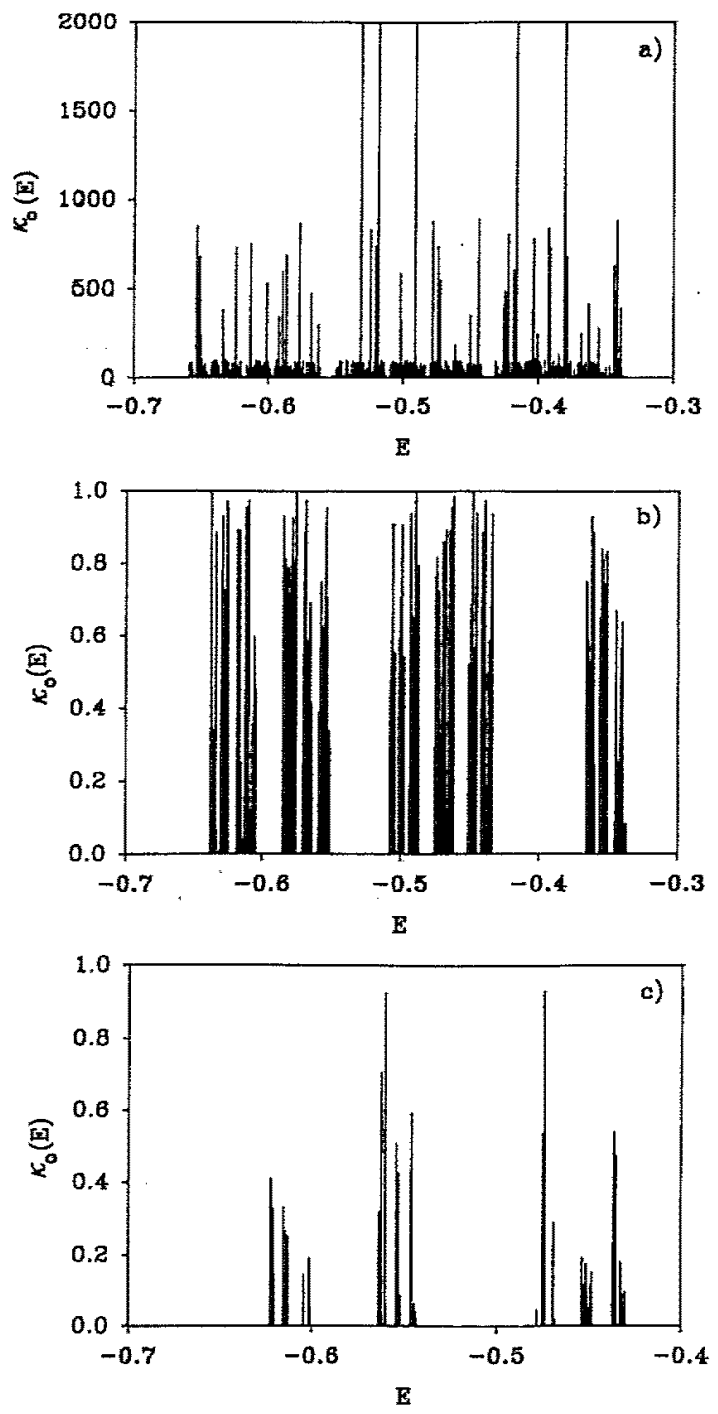

FIG. 3. Landauer conductance at zero temperature for a FSL with $N=987$ atoms and (a) $\alpha=0.99$, (b) 0.9 , and (c) 0.8 . 


$$
\kappa_{0}(E)=\frac{\tau(E)}{1-\tau(E)}
$$

The energy dependence of the transmission coefficient can be obtained in a straightforward manner in the transfer-matrix formalism. The calculation is carried out by embedding our finite FSL in an infinite periodic lattice of identical $A$ blocks. In this way we have calculated the Landauer conductance $\kappa_{0}$ for a wide range of model parameters $\alpha$ and $N$. A typical example of the obtained results is shown in Fig. 3 for $N=987$ and different values of the chemical diversity parameter. In the limit $\alpha \rightarrow 1$ the translational symmetry of the superlattice is recovered and, accordingly, it should be expected that Landauer conductance would diverge almost everywhere. On the contrary we observe in Fig. 3(a) that, for $\alpha=0.99$, the Landauer conductance exhibits a highly fragmented structure displaying dramatic fluctuations. Actually, there exists only a minor fraction of energy values where conductance reaches large values, whereas at the remaining energies it takes on a wide range of lower conductance values. This result clearly indicates the extreme sensibility of the conductance behavior to minute variations in the chemical composition of the FSL. Furthermore, note the overall decrease of the conductance peaks as the chemical diversity increases [compare the vertical scales of Figs. 3(a)-3(c)]. Finally, by comparing Fig. 3(a) with both 3(b) and 3(c), it becomes apparent that the fragmentation of the energy spectrum progressively manifests itself in the conductance pattern as $\alpha$ increases. Particularly, note the well-differentiated trifurcation structure of conductance peaks for $\alpha=0.9$ and $\alpha=0.8$. These features can be understood as follows. ${ }^{27}$ For any approximant to the FSL the allowed energies form a set of sparse points approximating a prefractal Cantor-set structure. As the electron energy equals one of these energy levels an enhanced resonant tunneling takes place leading to high conductance peaks. By increasing either the approximant length or the chemical diversity of the superlattice, its spectrum becomes more and more fragmented and strong fluctuations appear as a function of the electron energy. Therefore, the detailed structure of the energy spectrum naturally determines the finer details of the conductance pattern at zero temperature.

\section{FINITE-TEMPERATURE EFFECTS ON THE dC CONDUCTANCE}

In order to obtain realistic outcomes from the model, it is convenient to include in our study finite-temperature effects. To this end we shall consider the following expression, earlier discussed by Engquist and Anderson, for the dimensionless conductance of the system ${ }^{28}$

$$
\kappa(T, \mu)=\frac{\int\left(-\frac{\partial n}{\partial E}\right) \tau(E) d E}{\int\left(-\frac{\partial n}{\partial E}\right)[1-\tau(E)] d E}
$$

The integration is extended over the periodic $A$ superlattice band, $n$ is the Fermi-Dirac distribution, and $\mu$ denotes the chemical potential of the sample. We have calculated the expression (9) numerically using the transmission coefficient obtained in the previous section. The analysis of the obtained $\kappa(T, \mu)$ curves reveals interesting behaviors in both low- and high-temperature ranges. For convenience we consider these cases separately.

\section{A. de conductance at low temperatures}

In the limit $T \rightarrow 0$ the general form of the $\kappa(T, \mu)$ curve strongly depends on the adopted $\mu$ value. This is illustrated in Fig. 4 for a chain with $N=55$ and $\alpha=0.85$. We have observed the same conductance behavior, as that shown in Fig. 4, for larger approximants, up to $N=1597$. In Fig. 4(a) we show the transmission coefficient for this chain. The dashed vertical lines, labeled by roman numerals, indicate three different possible values for the chemical potential $\mu$. The corresponding conductance curves are plotted in Fig. $4(\bar{b})$. If the chemical potential is close to a set of transmission peaks, the conductance exhibits several characteristic humps. This is the case of curve I. Conversely, if the chemical potential is located in a main gap region, the conductance increases monotonically with temperature to reach a limiting value and no
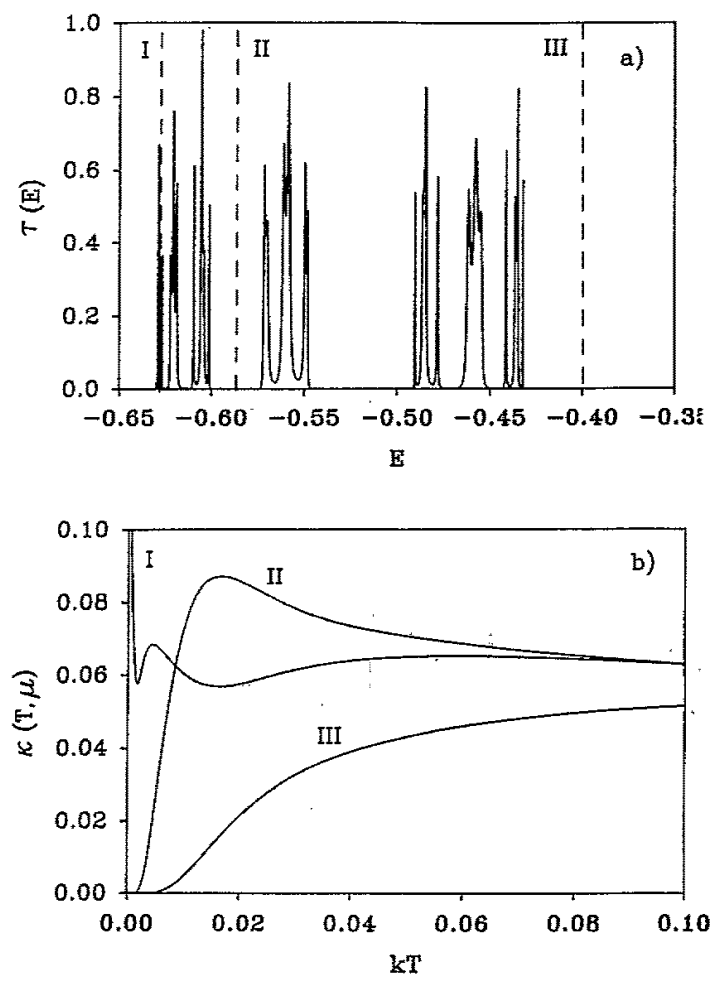

FIG. 4. Influence of the energy spectrum structure on the finite-temperature conductance of the FSL. In (a) we show the transmission coefficient for a chain with $N=55$ and $\alpha=0.85$. The vertical dashed lines indicate the position of the chemical potential in three different cases: (I) $\mu=-0.628$, (II) -0.585 , and (III) -0.4 . The corresponding conductance curves $\kappa(T, \mu)$ are shown in (b). 
relevant features are present at all, as seen in curve III. Finally, if the chemical potential lies in an intermediate region, a pronounced broad hump occurs due to the contribution of nearest transmission peaks. Such a hump is shown in curve II. Thus, it is clear that the occurrence of conductance humps at low temperatures is intimately related to the fragmented nature of the energy spectrum.

We will now prove that these different kinds of conductance behaviors can be related to the position of the chemical potential $\mu$ by means of a closed analytical expression. To this end we must consider the expression (9) in the low-temperature limit. It is well known that in this limiting case the curve $\kappa(T, \mu)$ is dominated by the weighting function $(-\partial n / \partial E)$ which appears in both integrands of (9). This function vanishes everywhere except for energies close to the $\mu$ value. For the sake of clarity let us assume that the chemical potential is located near a characteristic triplet of the transmission coeffcient. Now we introduce a further assumption by considering that the triplet structure can be roughly described in the form

$$
\tau(E) \simeq \sum_{i=1}^{3} \gamma_{i} \delta\left(E-E_{i}\right),
$$

where $\gamma_{i}$ is the characteristic strength of each transmission peak and $E_{i}$ denotes the position of the peak. Making use of expression (10) the conductance (9) can be approximated by

$$
\kappa(T, \mu) \simeq \frac{1+\cosh \beta \omega}{\sinh \beta \omega} \sum_{i=1}^{3} \gamma_{i} \beta n^{2}\left(\beta, E_{i}\right) e^{\beta \Delta E_{i}}
$$

where $\beta \equiv 1 / k T, \Delta E_{i} \equiv E_{i}-\mu$, and $\omega$ is the width of the triplet measured on the energy scale. Thus, once the chemical potential has been fixed, the evolution of the conductance curve is determined by four basic parameters: the width $\omega$ and the transmission strengths $\gamma_{i}$. The prefractal nature of the spectrum implies that the triplet width depends on the hierarchical level considered so that one may expect different conductance features to appear at different energy scales. In Fig. 5 we plot the conductance curve for a FSL whose chemical potential is located inside a characteristic triplet of the energy spectrum. The curve displays two different behaviors depending on the considered temperature scale. At very low temperatures $(k T \sim 0.005)$, the curve exhibits a prominent hump which displays finer structure. The inset shows the dependence of this structure on small variations of the sample chemical potential. The occurrence of the spike in the conductance hump is directly related to the proximity of $\mu$ to the transmission peak located at $E=-0.5484$. On the other side, at higher temperatures $(k T \sim 0.05)$, the conductance curve shows a very broad maximum, due to the contribution of the $a$ main subband as a whole. As we see, the influence of the spectrum structure on the finite-temperature conductance is rather significative. Most interestingly, we can account for most details appearing in the conductance curve by means of the analytical expression (11). As an example, in Fig. 6 we compare the conductance curve of Fig. 5, which has been obtained numerically from the expression (9), with that corresponding to the analytical expression (11) when suitable parameters for $\omega$ and $\gamma_{i}$ are introduced. The agreement between both curves is excellent, especially if one considers that each peak appearing in the considered triplet has a well-developed inner structure and the $\delta$-function approach may result rather crude. We wish to stress again that the parameters $\omega$ and $\gamma_{i}$ used to evaluate expression (11) above completely de-

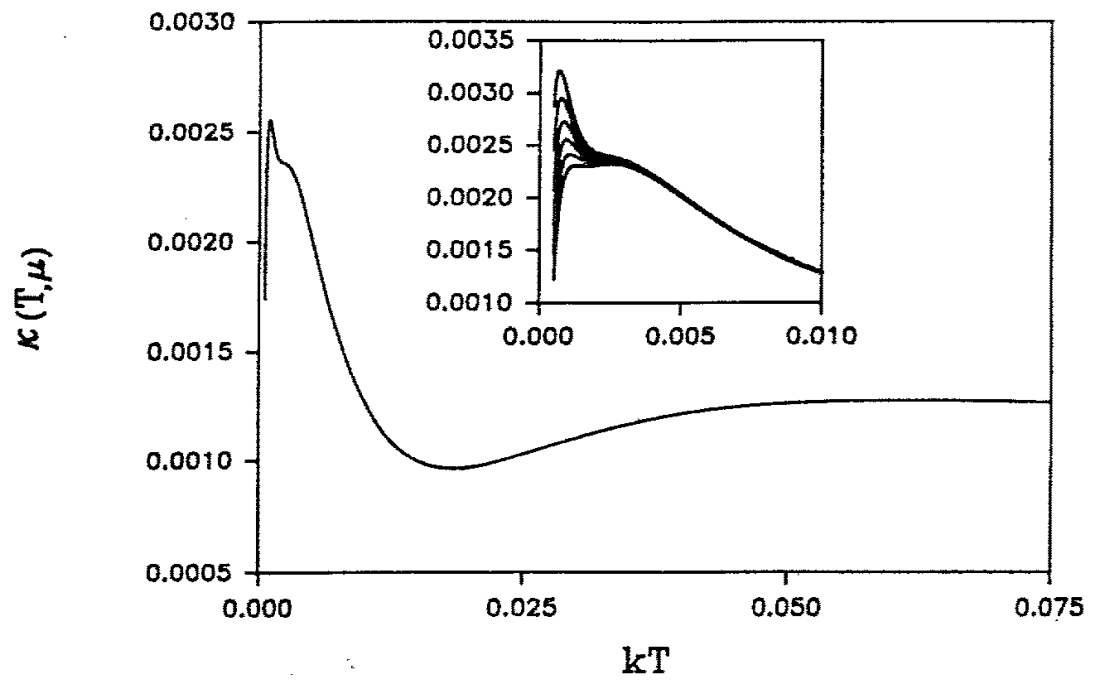

FIG. 5. Conductance curve versus temperature for a FSL with $N=987, \alpha=0.75$, and $\mu=-0.5498$. The inset gives a close view of the dependence of the hump structure on the adopted chemical potential value. From top to bottom the energies corresponding to the different curves range from $\mu=-0.5495$ to $\mu=-0.5500$ with a step of 0.0001 . Note that the spike shifts to higher temperatures as the chemical potential separates from the transmission peak located at $E=-0.5484$. 


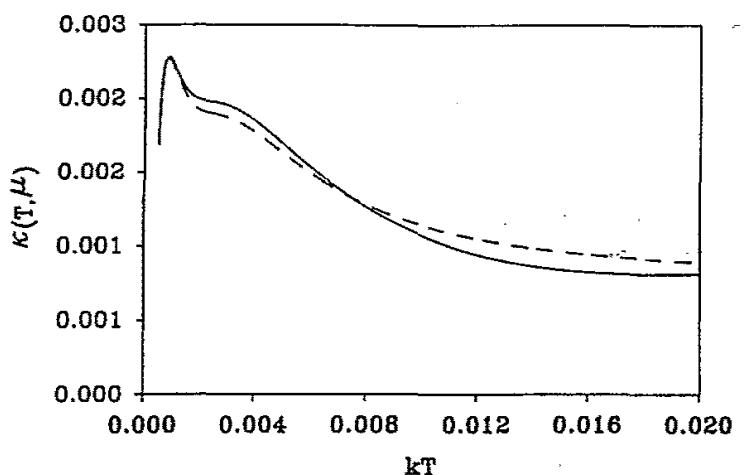

FIG. 6. Comparison between the conductance obtained from the exact expression (solid line) and that obtained from the approximate analytical expression (11) (dashed line) with $\mu=-0.5493, \omega=0.028, \gamma_{1}=1.085, \gamma_{2}=1.000$, and $\gamma_{3}=1.533$.

termine the energy spectrum of the FSL. In consequence, the analytical expression (11) could provide a very useful tool to fit experimentally measured conductance curves in order to obtain from them relevant parameters describing the underlying energy spectrum structure, especially those determining the fragmentation splitting pattern. We have numerically checked that the validity of the expression (11) can be extended to higher fragmentation levels of the energy spectrum up to the third splitting stage. Hence, we can confidently conclude that, expression (11) allows for an excellent characterization of the influence of the highly fragmented nature of the FSL energy spectrum on the dc conductance at low temperatures.

\section{B. dc conductance in the high-temperature limit}

In our numerical study we have observed that the $\kappa(T, \mu)$ curves rapidly saturate reaching stable asymptotic values at about $k T \simeq 0.5$ for all the values of $\alpha$ and $N$ considered in our study. Note that this value is of the order of the $A$ perfect superlattice minibandwidth $\Omega=4 e^{-d}$. Moreover, the asymptotic regime does not depend on the position of the chemical potential at all. This fact can be explained as follows. Let $\Delta E \equiv E-\mu$; hence for $\beta \Delta E \ll 1$ we can expand the weighting function as

$$
-\frac{\partial n}{\partial E} \simeq \frac{1}{4}+O\left[(\beta \Delta E)^{2}\right]
$$

Since $\Delta E<\Omega$ for every electron energy we consider, the expansion above becomes valid whenever the temperature satisfies the condition $k T>\Omega$. In this case expression (9) can be approximated by.

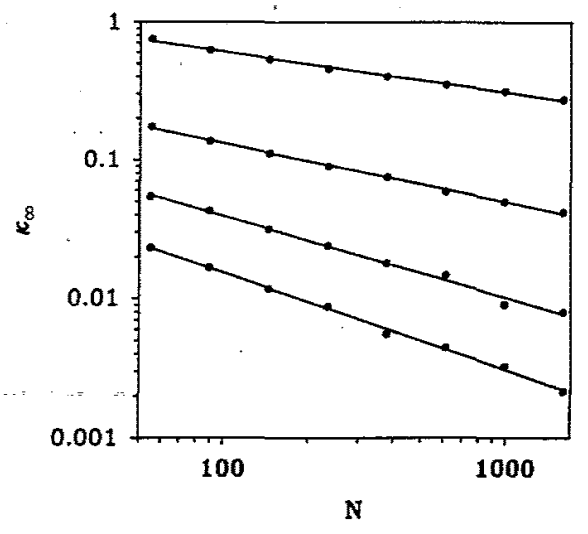

FIG. 7. Dependence of the high-temperature limit conductance (dots) on the sample length for several values of the chemical diversity parameter. From top to bottom these values of $\alpha$ are $0.95,0.90,0.85$, and 0.80 . The solid lines give the least-squares fits describing a power-law behavior. The scaling exponents are respectively given by $-0.2968,-0.4245$, -0.5887 , and -0.6986 .

$$
\kappa(T, \mu) \simeq \frac{\int \tau(E) d E}{\int[1-\tau(E)] d E}, \quad \beta \rightarrow 0,
$$

where the integration extends over the allowed band. Note that expression (13) only depends on the model parameters $\alpha$ and $N$ through the transmission coefficient $\tau(E)$. In Fig. 7 we show the behavior of the asymptotic dc conductance of the FSL with the sample size for several values of the parameter $\alpha$. This plot clearly reveals that, in the high-temperature limit, the conductance of the FSL scales with the chain length according to a power law, whose exponent strongly depends on the chemical diversity of the superlattice.

\section{Discussion of the obtained results}

The conductance behavior in the high-temperature limit can be explained as due to the fact that when $k T$ equals the perfect superlattice minibandwidth $\Omega$, all electrons contribute to the electronic transport in the superlattice growth direction. This result qualitatively agrees with time-resolved photoluminescence spectra obtained for GaAs/GaAlAs FSL's (Ref. 16) and allows us to estimate the temperature range for which our predicted conductance humps may be observable. In fact, typical bandwidths for periodic superlattice minibands are about $100 \mathrm{meV}$. Therefore, as can be seen from Fig. 4(b), the high-temperature limit will be achieved for temperatures of about $T=1 / 10 k \approx 1100 \mathrm{~K}$. As a consequence, conduction humps due to the first level of fragmentation should be observable at $T \approx 200 \mathrm{~K}$ and conduction spikes due to the third level of splitting should be predominant at temperatures below $T \approx 20 \mathrm{~K}$. These temperature ranges are easily accessible to actual experimental arrangements, hence indicating the possibility of experimental observation of the prefractal spectrum structure in finite approximants to the Fibonacci lattice. 


\section{CONCLUSIONS}

In summary, we have studied a general system describing a 1D superlattice in which the constituent blocks are arranged according to the Fibonacci sequence. To this end we have derived a tight-binding description from a simple, continuous Schrödinger equation which allows us to explicitly take into account the interaction between nearest-neighbor blocks. The resulting energy spectrum has been obtained by means of the usual transfer-matrix techniques for a wide variety of possible realizations. The obtained spectra show typical features of quasiperiodic systems: The spectrum appears highly fragmented and displays a well-developed self-similar structure characteristic of a prefractal Cantor-like set. Another typical signature of quasiperiodic systems corresponds to its spectral measure which can be roughly characterized by means of the equivalent bandwidth of the spectrum. Our results corroborate the generalized view supporting that almost all eigenstates are critical in Fibonacci chains. From the analysis of the equivalent bandwith dependence on the chemical diversity parameter $\alpha$, we have been able to conclude that the existence of mobility edges characterizing a metal-insulator transition are not to be expected in this kind of superlattice. However, the chemical composition of the superlattice has an interesting effect on the global structure of the spectrum; i.e., it very precisely determines both the position and widths of the main subbands and gaps. Hence, one can think about the possibility of very accurately determining the electronic structure of the sample by usual computer controlled doping deposition techniques. The electronic structure of the energy spectrum is naturally translated to the magnitudes describing transport properties of the superlattice through the transmission coefficient. As has been fully discussed in Sec. $V$, when the chemical poten- tial is located near a transmission peak, the dc conductance of the FSL experiences a pronounced hump at low temperatures. Therefore, we can determine the electronic transport properties of the system at low temperatures by properly selecting those parameters which characterize it: its chemical composition and sample length. This interesting result reveals the possibility of a certain degree of "engineering" of transport properties during sample growth. Such a possibility can be of considerable interest in order to construct superlattice heterostructures exhibiting fine-tuning capabilities. As regards to conductance at high temperatures, we have found that it reaches an asymptotic regime for $k T$ values of order of the perfect superlattice minibandwidth. The asymptotic value scales with the system size, and the characteristic exponent only depends on the chemical diversity parameter $\alpha$. Since $\alpha$ also determines the hierarchical splitting of the electronic spectrum, it would be then possible to estimate the fragmentation degree of the spectrum by measuring the conductance of samples with different lengths. Finally, we remark that an interesting issue emerging from this work concerns the possibility of using the analytical expression (11) in order to obtain significant information about the fragmentation of the electronic spectrum in quasiperiodic superlattices from experimentally measured conductances at low temperatures. To the best of our knowledge this is a novel result which could be easily applied as soon as suitable measures are available.

\section{ACKNOWLEDGMENTS}

A.S. is partially supported by DGICyT (Spain) through Project No. PB92-0248, and by European Union through NETWORK nonlinear Spatio-Temporal Structures in Semiconductors, Fluids, and Oscillator Ensembles.
* Also at the Instituto de Estudios Interdisciplinares, El Guijo, Z4 Galapagar, E-28260 Madrid, Spain.

${ }^{1}$ M. Kohmoto, L. P. Kadanoff, and C. Tang, Phys. Rev. Lett. 50, 1870 (1983).

${ }^{2}$ S. Ostlund and R. Pandit, Phys. Rev. B 29, 1394 (1984).

${ }^{3}$ S. N. Karmakar, A. Chakrabarti, and R. K. Moitra, J. Phys. Condens. Matter 1, 1423 (1989).

${ }^{4}$ G. Y. Oh, C. S. Ryu, and M. H. Lee, J. Phys. Condens. Matter 4, 8187 (1992).

${ }^{5}$ M. Severin and R. Riklund, Phys. Rev. B 39, 10362 (1989).

${ }^{6}$ Y. Kim, M. H. Lee, and M. Y. Choi, Phys. Rev. B 40, 2581 (1989).

7 R. Merlin, K. Bajema, R. Clarke, F. -Y. Juang, and P. K. Bhattacharya, Phys. Rev. Lett. 55, 1768 (1985).

${ }^{8}$ J. Todd, R. Merlin, R. Clarke, K. M. Mohanty, and J. D. Axe, Phys. Rev. Lett. 57, 1157 (1986).

${ }^{2}$ F. Laruelle and B. Etienne, Phys. Rev. B 37, 4816 (1988).

${ }^{10}$ M. Nakayama, H. Kato, and S. Nakashima, Phys. Rev. B 36, 3472 (1987).

${ }^{11}$ K. Kono, S. Nakada, Y. Narahara, and Y. Ootuka, J. Phys. Soc. Jpn. 60, 368 (1991).

12 D. Tuet, M. Potemski, Y. Y. Wang, J. C. Maan, L. Tapfer, and K. Ploog, Phys. Rev. Lett. 66, 2128 (1991).

${ }^{13}$ A. Chakrabarti, S. N. Karmakar, and R. K. Moitra, Phys.
Lett. A 168, 301 (1992).

${ }^{14}$ P. Hawrylak and J. Quinn, Phys. Rev. Lett. 57, 380 (1986).

${ }^{15} \mathrm{H}$. Hiramoto and M. Kohmoto, Phys. Rev. Lett. 62, 2714 (1989).

${ }^{16}$ A. Yamaguchi, T. Saiki, T. Ninomiya, K. Mísawa, and T. Kobayashi, Solid State Commun. 75, 955 (1990).

${ }^{17}$ S. Katsumoto, N. Sano, and S. Kobayashi, Solid State Commun. 85, 223 (1993).

${ }^{18}$ V. Kumar, J. Phys. Condens. Matter 2, 1349 (1990).

19 F. Domínguez-Adame, J. Phys. Condens. Matter 1, 109 (1989).

${ }^{20}$ P. Erdös and R. C. Herndon, Helv, Phys. Acta 50, 513 (1977).

${ }^{21}$ R. E. Borland, Proc. R. Soc. London Ser. A 274, 529 (1963).

23 J. B. Sokoloff, Phys. Rep. 126, 189 (1985).

${ }^{23} \mathrm{Y}$. Liu and R. Riklund, Phys. Rev. B 35, 6034 (1987).

${ }^{24}$ Y. Liu and W. Sritrakool, Phys. Rev. B 43, 1110 (1991).

${ }^{25}$ N. Niu and F. Nori, Phys. Rev. Lett. 57, 2057 (1986); Phys. Rev. B 42, 10329 (1990).

${ }^{28}$ R. Landauer, IBM J. Res. Dev. 1, 223 (1957).

${ }^{37}$ S. Das Sarma and X. C. Xie, Phys. Rev. B 37, 1097 (1988).

${ }^{28}$ H. L. Engquist and P. W. Anderson, Phys. Rev. B 24, 1151 (1981). 